BMJ Open

Diabetes

Research

\& Care

\title{
Intent-to-treat analysis of a simultaneous multisite telehealth diabetes prevention program
}

\author{
Elizabeth L Ciemins, ${ }^{1,2}$ Patricia J Coon, ${ }^{1}$ Nicholas C Coombs, ${ }^{1}$ \\ Barbara L Holloway, ${ }^{1}$ Elizabeth J Mullette, ${ }^{1}$ William N Dudley ${ }^{3}$
}

To cite: Ciemins EL, Coon PJ, Coombs NC, et al. Intent-totreat analysis of a simultaneous multisite telehealth diabetes prevention program. BMJ Open Diab Res Care 2018;6:e000515. doi:10.1136/ bmjdrc-2018-000515

ELC and PJC contributed equally.

Received 19 January 2018 Revised 29 March 2018 Accepted 4 April 2018

\section{Check for updates}

${ }^{1}$ Center for Clinical Translational Research, Billings Clinic, Billings, Montana, USA ${ }^{2}$ AMGA (formerly American Medical Group Association), Alexandria, Virginia, USA ${ }^{3}$ Department of Public Health Education, University of North Carolina-Greensboro, Greensboro, North Carolina, USA

Correspondence to Dr Elizabeth L Ciemins; eciemins@amga.org

\section{ABSTRACT}

Objective Determine the effectiveness of a 16-week modified diabetes prevention program (DPP) administered simultaneously to multiple rural communities from a single urban site, as compared with a similar face-to-face intervention. A 12-week intervention was evaluated to consider minimization of staff costs in communities where resources are limited.

Research design and methods A prospective cohort study compared DPP interventions implemented in rural (via telehealth technology) and urban (face-to-face) communities using an intent-to-treat analysis. Primary outcome measures included $5 \%$ and $7 \%$ body weight loss. Logistic regression analyses were used to determine predictors of intervention success and included a variable for treatment effect.

Results Between 2010 and 2015, up to 667 participants were enrolled in the study representing one urban and 15 rural communities across Montana. The 16-week urban and rural interventions were comparable; $33.5 \%$ and $34.6 \%$ of participants lost $7 \%$ body weight, respectively; $50 \%$ and $47 \%$ lost $5 \%(p=0.22)$. Participants who were male $(\mathrm{OR}=2.41 ; 95 \% \mathrm{Cl} 1.32$ to 4.40$)$, had lower baseline body mass index ( $0 \mathrm{R}=1.03 ; 95 \% \mathrm{Cl} 1.01$ to 1.07$)$, attended more sessions ( $\mathrm{OR}=1.33 ; 95 \% \mathrm{Cl} 1.11$ to 1.58 ), and more frequently reported $(\mathrm{OR}=3.84 ; 95 \% \mathrm{Cl} 1.05$ to $14.13)$ and met daily fat gram $(0 \mathrm{R}=4.26 ; 95 \% \mathrm{Cl} 1.7$ to $10.6)$ and weekly activity goals $(\mathrm{OR}=2.46 ; 95 \% \mathrm{Cl} 1.06$ to 5.71) were more likely to meet their $7 \%$ weight loss goal. Predictors of meeting weight loss goals were similar for participants enrolled in the 12-week intervention. Conclusions Using telehealth technology to administer a modified DPP to multiple rural communities simultaneously demonstrated weight loss results comparable to those in a face-to-face intervention. Given the limitation of resources, linking rural areas to urban centers using telemedicine may increase access to much needed services to prevent or delay progression to diabetes.

Since the first publication of its success in 2002, the diabetes prevention program (DPP) has demonstrated both efficacy and effectiveness in reducing the progression to type 2 diabetes mellitus in both laboratory and real-world settings. ${ }^{12}$ Sixteen years later, the Center for Medicare and Medicaid Services (CMS) is planning to pay for face-to-face services with

\section{Significance of this study \\ What is already known about this subject? \\ - Numerous studies support the effectiveness of the diabetes prevention program (DPP) in the prevention of progression from pre-diabetes to type 2 diabetes mellitus when delivered in a group face- to-face setting. \\ What are the new findings? \\ - This study demonstrates the effectiveness of delivering the DPP via telehealth technology simultaneously to multiple remote sites from one location. \\ - No differences were observed between face-to- face and telehealth DPP. \\ How might these results change the focus of research or clinical practice? \\ - These results have the potential to significantly impact clinical practice as telehealth DPPs can be used to reach patients who lack access to the DPP due to geographic distance or lack of a local program. \\ - In addition, this study provides the necessary evidence for payers to reimburse for DPPs administered using telehealth technology, providing much needed services to individuals living in rural communities or any setting who lack access to DPPs.}

a new physician fee schedule in calendar year $2018,{ }^{3}$ but they are not yet covering telehealth-delivered DPP. The landmark DPP trial, conducted in 27 clinic sites across the USA, found that modest weight loss through lifestyle modification, that is, dietary changes and increased physical activity, was better than treatment with metformin in preventing or delaying the onset of type 2 diabetes among at-risk patients. ${ }^{2}$ Specifically, the incidence of new-onset diabetes was reduced by $58 \%$ with intensive lifestyle intervention and by $31 \%$ with metformin, as compared with placebo. With one case of diabetes prevented for every seven persons treated for 3 years, lifestyle 
modifications have been shown to be highly effective means of delaying or preventing type 2 diabetes $^{2}$ and by association, its complications. Delaying or preventing complications of diabetes, for example, foot amputations, retinopathy, neuropathy, nephropathy, cardiovascular disease, and vascular dementia, has the potential to reduce the individual, health system, and societal health burden of diabetes. ${ }^{45}$

Prompted by the success of the national DPP, modified DPP studies using group-based face-to-face delivery models have demonstrated comparable findings to those achieved in the original DPP study. ${ }^{6-16}$ In 2008 , investigators in one rural state implemented a modified groupbased face-to-face DPP in a small community ${ }^{17}$ and by 2016, similar programs had been implemented across the state. ${ }^{6}$ Although the implementation of the modified DPP delivery model across the state enabled many patients to benefit, access issues remained, as these programs were primarily offered in larger towns and cities, leaving those in small, rural communities without similar opportunities.

To address this challenge, since 2009 one rural Montana health care organization has been administering a modified group DPP simultaneously to both an on-site group and a second small group in a remote frontier community via telehealth videoconferencing, and found comparable outcomes to the original DPP study. ${ }^{815} \mathrm{~A}$ Pittsburgh, PA study (the TREAT model-Telemedicine for Reach, Education, Access, and Treatment) reported improved diabetes-related behavioral and psychosocial outcomes as well as high patient satisfaction when providing one-on-one diabetes self-management education via videoconferencing to participants in rural areas in $2014 .{ }^{18}$ While the TREAT and other studies ${ }^{19}$ have demonstrated the effective delivery of diabetes education and services via telehealth, the current study is only the second study that has examined the effectiveness of using telehealth technology to deliver the DPP. Although Vadheim et $a \ell^{81517}$ demonstrated telehealth-delivered DPP effectiveness in one community connected to an urban setting, our study presented in this manuscript is the first example of delivering a program simultaneously to multiple rural and frontier sites, addressing the important gap in care present in rural communities with limited resources. Additionally, a systematic review of program modification strategies of the DPP found no significant differences in weight or body mass index (BMI) reduction associated with any type of modification of the DPP. ${ }^{20}$ They did find, however, that programs with a maintenance component, similar to the current study, achieved greater mean weight loss.

Using an intent-to-treat (ITT) approach, the objective of this study was to determine the effectiveness of a 16-week modified group-based DPP administered simultaneously to multiple rural communities from a single urban community, an educational method that has not yet been studied. Additionally, a 12-week program was evaluated to consider a version that would potentially minimize staff costs in rural communities where resources are particularly limited. In a country where one in three people are affected by pre-diabetes, expansion of the DPP is crucial, including innovative modes of delivery, such as telehealth, with broad reach to many communities simultaneously. This research has the potential to provide evidence in support of coverage for these services, delivered via telehealth, by CMS and other payers. Thus, CMS' physician reimbursement rule that goes into effect in 2018 could be extended to cover telehealth-delivered DPP based on this research.

\section{RESEARCH DESIGN AND METHODS}

\section{Study design and setting}

This observational study compared a modified DPP delivered via telehealth technology in 15 unique rural communities with a DPP delivered face-to-face in one urban community from 2010 to 2015 (9 were part of Rural-16; 14 were part of Rural-12; 8 were part of both). To ensure generalizability, the modified DPP adhered closely to the Centers for Disease Control and Prevention (CDC) standards for DPP recognition including use of the CDC-approved curriculum, participant eligibility, acquisition of data reflecting participant progress, safety and privacy of participant data, trained lifestyle coaches, and designated DPP coordinators. In addition, the 'reach' was broad to 15 rural communities in 14 counties, who all agreed to participate, indicating general acceptance and willingness to participate across the state. County populations ranged from 1102 to 13282 , with a mean population of 2.64 persons per square mile (range $0.3-5.1$ ); counties were located 35-356 miles from the urban hub.

The primary objective of this ITT analysis was to determine whether an intervention delivered simultaneously to multiple communities via telehealth resulted in clinical outcomes comparable to an intervention delivered face-to-face. As an ITT analysis, all patients enrolled in the study were included in the analysis, regardless of attendance. Primary outcomes included weight loss of $\geq 7 \%$ and $\geq 5 \%$. Secondary outcomes included participants meeting the following goals: engaging in at least 150 minutes of moderate physical activity per week and meeting individually defined daily fat gram intake goals. Participant attendance was also tracked. Attrition rates, or the percentage of patients who dropped out of the program, were recorded. These patients are considered 'lost to follow-up,' which refers to patients who were once participating in a study but have become lost, or unreachable, at the point of follow-up. The program was modified enabling its delivery via telehealth technology. In addition, predictors of success across all program types were identified.

\section{Intervention}

Over the course of the 6-year study period, three DPP interventions were implemented: a 16-week urban, face-to-face program (Urban-16); a 16-week rural, telehealth program 
(Rural-16); and a 12-week rural, telehealth program (Rural-12). Urban-16 and Rural-16 programs comprised 16 weekly sessions (the core period of the intervention), followed by sixmonthly or bimonthly group sessions (the postcore period of the intervention). The Rural-12 program, which included all of the same content as Rural16, but with four sessions condensed and combined, comprised 12 weekly sessions representing the core period, followed by sixmonthly or bimonthly group sessions, representing the postcore period. In the Urban-16 intervention, the intervention sessions were led by a registered dietitian except for two sessions led by an exercise physiologist and two by a licensed clinical social worker. The Rural-16 and Rural-12 intervention sessions were taught by an interdisciplinary team consisting of a dietitian, nurse certified diabetes educator, health coach (social worker) and physical therapist/exercise physiologist. The interdisciplinary team was located at the urban hub. There was significant overlap between the staff teaching the rural and face-to-face participants, however the lead dietitians were different individuals. Close attention was paid to maintain the fidelity between the two programs, including frequent communication between the lead dietitians. In both rural interventions, the urban hub focused solely on the rural participants; there were no face-to-face participants.

In each of the three interventions or programs, several DPP classes were conducted each year from 2010 to 2015. Each class comprised either 12 or 16 core sessions, followed by a postcore period. Twelve Urban- 16 face-toface group classes were held at one urban site with 9-23 participants per class. Six Rural-16 and eight Rural-12 telehealth group classes were dispensed from one urban health care organization hub to 15 rural communities. In any given class, five to eight rural communities were simultaneously connected to the urban hub; 35-48 participants were in attendance with 4-24 participants per community. Rural community classes were held in their local health care clinics. Each rural site selected an individual from the local clinic staff to serve as their site coordinator. Coordinators were responsible for recruiting participants, collecting data, and moderating the DPP sessions held in their community. Site coordinators were critical to the success of the intervention as a liaison between the urban site interdisciplinary team and local primary care providers.

Throughout the DPP intervention classes, participants were asked to keep a diary in which they tracked daily fat grams consumed and minutes of physical activity performed. At each DPP session, site coordinators collected this information and obtained a participant body weight and an initial height. After each session, data were faxed to the urban site team who reviewed and commented on each participant's self-reported fat intake and physical activity levels and provided feedback directly to participants. (In 2015, this method was updated to an electronic communication system.) Content of the modified DPP sessions taught during each class was based on the national DPP guidelines. ${ }^{21}$
In this study, telehealth technology was administered by the Eastern Montana Telemedicine Network, which is housed at Billings Clinic in Billings, MT and has 42 partners in 29 rural and frontier communities in Montana, Wyoming, and North Dakota. The telehealth DPP (Rural-16 and Rural-12) was delivered in real time from the urban hub to rural sites simultaneously using Polycom videoconference units via dedicated broadband or T1 lines. The urban hub site also used computers and electronic document stands to facilitate transmission of videos, PowerPoint presentations, and other educational documents. Telehealth connections were secure and compliant with the Health Insurance Portability and Accountability Act (HIPAA).

\section{Participant eligibility}

Program eligibility criteria included patients with BMI $>24 \mathrm{~kg} / \mathrm{m}^{2}$; a diagnosis of pre-diabetes (International Classification of Diseases Ninth Revision (ICD-9) 790.29, ICD-10 R73.03) or at least one cardiovascular risk factor; and the physical ability to participate in the exercise component of the program. For rural sites to participate, rural communities had to have teleconferencing capability in their local health care facility that could connect to the urban health care organization.

\section{Outcome measures}

Outcomes were compared between Urban-16 and Rural16. Due to the shortened length of Rural-12, this intervention group was evaluated separately using a pre/post comparison. Four primary binary dependent variables for weight loss goals were examined in this study and included: (1) $7 \%$ body weight loss in core period of the intervention; (2) $5 \%$ body weight loss in core period; (3) $7 \%$ body weight loss at any time during either the core or postcore periods; and (4) $5 \%$ body weight loss at any time during either the core or postcore periods. The study investigators included a $5 \%$ weight loss goal (in addition to the $7 \%$ weight loss goal) because studies conducted to support the DPP demonstrated body weight loss of $5 \%$ to be protective in the progression to type 2 diabetes. ${ }^{2}$ Independent variables included patient demographics and baseline clinical measures, that is, age, weight, BMI, and sex. Intermediate outcomes of attendance during core and postcore DPP sessions, and recording and meeting daily fat gram and weekly physical activity goals, were also examined as predictors of intervention success.

\section{Statistical analysis}

Two primary analyses were conducted: (1) a comparison of Urban-16 with Rural-16 using an ITT approach; and (2) analysis of predictors of intervention success across both groups. In addition, a comparison with the longer interventions, that is, 16 weeks, as well as a logistic regression predictive analyses for Rural-12 were conducted separately. The authors hypothesized that there would be no significant difference between the urban and rural interventions. In the first analysis, Student's t-tests 
were used to evaluate differences for continuous variables and $\mathrm{X}^{2}$ or Fisher's exact test was used to evaluate differences for categorical variables. For both analyses, multivariable logistic regression models were constructed for each outcome to estimate fit and model strength. Participants in 16-week and 12-week interventions were modeled separately. Following an ITT approach, no participant was dropped from the analyses. If a participant attended only one session, the measures collected at that single visit were used for both preintervention and postintervention values, that is, no change in weight was recorded. To enable comparison to DPP studies in the literature, the first analysis was also done on a subset of participants who were engaged in the program, defined as having attended one of the last three core sessions and at least one of the postcore sessions. There is not a single definition for completion or engagement. To become a CDC-recognized program, 60\% of participants are required to attend at least nine core sessions and three postcore sessions ${ }^{22}$ and participants in this study met this definition. Our definition of engagement aligns with other studies that have defined completion as 'logged in within last 30 days of intervention, ${ }^{, 14}$ or 'had a laboratory measurement during after-core'. ${ }^{23}$

Overall model fit was assessed with the Nagelkerke ${ }^{24}$ pseudo $\mathrm{R}^{2}$ and results were presented as ORs. Collinearity was assessed by reviewing correlations and variance inflation factors. ${ }^{25} \mathrm{~A}$ backwards stepwise regression analysis was conducted and included independent variables with the strongest loadings. All analyses were performed using SAS statistical software V.9.4 (SAS Institute) and SPSS V.22.0 (IBM. Released 2013. IBM SPSS Statistics for Windows). Two-sided tests were conducted at the $\alpha=0.05$ level.

\section{Power}

When initially composed, sample size calculations for each intervention were determined based on hypothesis testing from the primary research question and used preliminary data to determine ranges and variances. Each individual study was adequately powered based on the unique parameters for that study. It follows that there was sufficient power for the current study as it was adequately calculated in parent studies. Additionally, there were no significant differences in attrition (figure 1) that would undermine the level of power within this study. A post hoc power analysis further confirmed this determination.

\section{RESULTS}

\section{Study enrollment}

A total of 667 participants were recruited from one urban and 15 rural Montana communities from 2010 to 2015 to participate in one of three modified DPP intervention groups: Urban-16, Rural-16, and Rural-12, as shown in figure 1. Class size ranged from 9 to 48 with 4-24 participants per site. Attrition rates were similar across groups with $34 \%-37 \%$ lost to follow-up before the end of the core sessions, and of those who completed the core period, 22\%-29\% were lost to follow-up before the end of the postcore period. However, following a strict, ITT approach, all patients were maintained in the study data set, regardless of attendance. The mean percentage of enrolled participants who completed an entire class, that is, all 12 or 16 sessions, ranged from $45 \%$ to $51 \%$ (Urban16: 51\%; Rural-16: 45\%; Rural-12: 51\%). Participants in all three groups attended on average more than $65 \%$ of all class sessions $(65 \%-71 \%$ of the core sessions and $23 \%-31 \%$ of postcore sessions). As can be seen in table 2, the mean number of core plus postcore sessions attended was 13.0, 12.2, and 9.6 for Urban-16, Rural-16, and Rural12 , respectively.

\section{Telehealth DPP effectiveness}

Table 1 compares baseline characteristics of the three study groups: Urban-16, Rural-16, and Rural-12. Forty-one per cent of total participants were in the Urban-16 group. Nearly equal percentages participated in Rural-16 and Rural-12, $28.6 \%$ and $29.7 \%$, respectively. Participants were predominantly female $(83 \%-$ $88 \%$ ) and mean age ranged from 51.8 to 55.7 years across groups. Mean BMI ranged from 37.0 to $37.6 \mathrm{~kg} /$ $\mathrm{m}^{2}$. There were no significant differences in participant characteristics across the three groups.

\section{Comparison of Urban-16 with Rural-16}

Table 2 shows that the Urban- 16 and Rural-16 interventions were comparable across all weight loss outcome goals. Using an ITT approach, the percentage of Urban-16 and Rural-16 participants who met their $7 \%$ weight loss goal overall was $33.5 \%$ and $34.6 \% \quad(p=0.22)$, respectively; those who met their $5 \%$ weight loss goal were $49.6 \%$ and $46.6 \%(\mathrm{p}=0.22)$. Secondary lifestyle activity outcomes were also comparable except for the percentage of time weekly physical activity minutes' goals ( $150 \mathrm{~min} /$ week) were met; Rural-16 participants reported meeting their goal more often (Rural-16: $35.1 \%$ of time vs Urban16: $25.8 \%$ of time $(p<0.05))$. Per cent and total weight loss were also comparable between Rural-16 (8.9\%, 6.6 $\mathrm{kg})$ and Urban-16 $(8.8 \%, 6.5 \mathrm{~kg})$ participants $(\mathrm{p}>0.05)$. (Rural-12 participants who demonstrated $5.1 \%$ weight loss of a mean $3.7 \mathrm{~kg}$ are included in table 2 for descriptive comparison purposes only; no statistical tests were conducted to evaluate differences between Rural-12 and the interventions with longer core periods.)

In addition to the ITT study population, results from a subset of participants who completed their respective classes were also calculated and are included in table 2. The percentage of Urban-16 and Rural-16 participants who met their $7 \%$ weight loss goal overall was $46.2 \%$ and $52.1 \%(\mathrm{p}=0.32)$, respectively; those who met their $5 \%$ weight loss goal were $65.8 \%$ and $70.3 \%(\mathrm{p}=0.41)$.

\section{Predictors meeting weight loss goals}

Table 3 displays the predictors of participants' success by combining the 16-week interventions. Participants who 
Treatment Group Enrollment

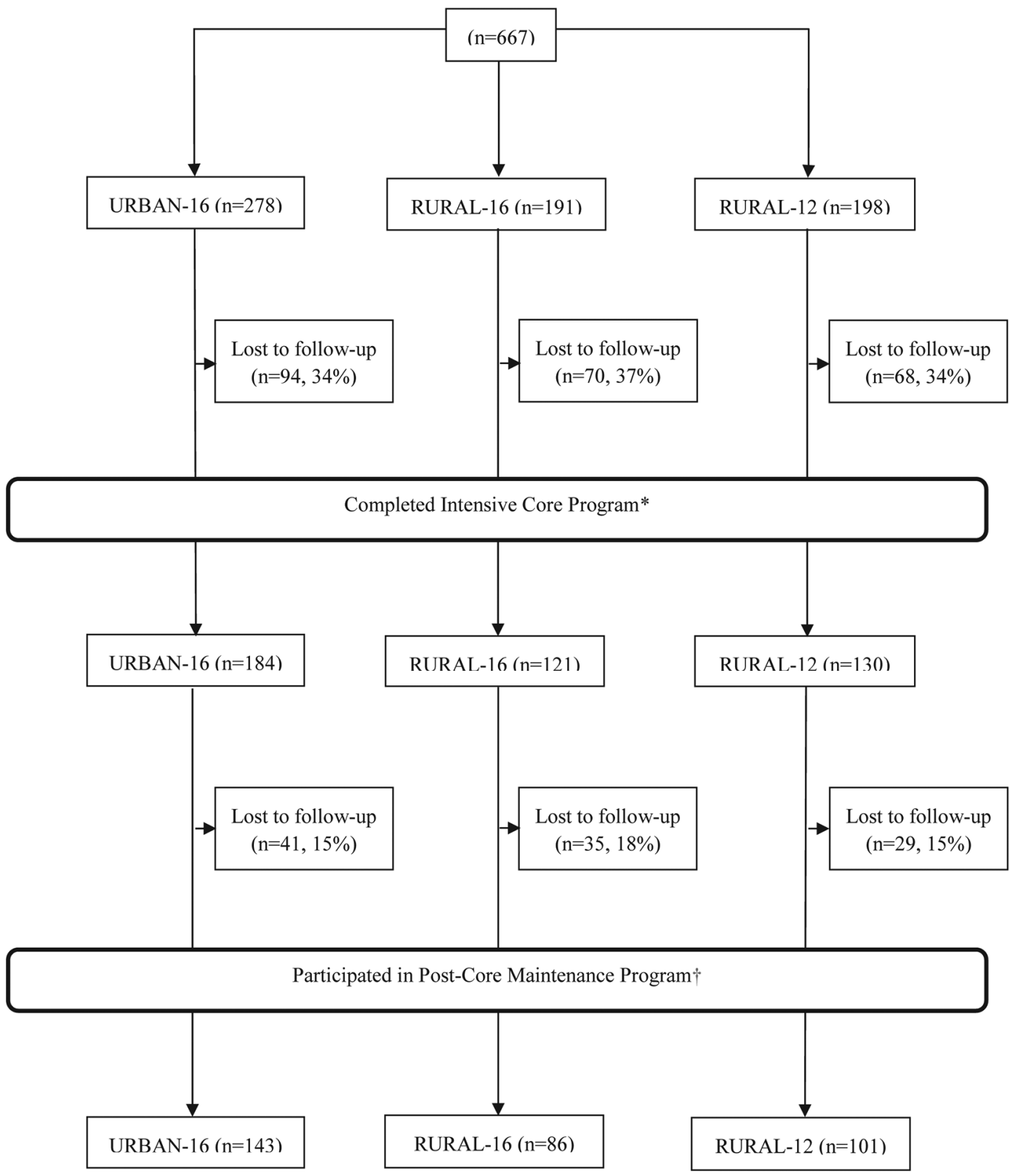

* Completion of Intensive Core Program defined as attendance at any of last three sessions.

$\uparrow$ Participation in Post-core Maintenance Program defined as attending at least one session.

Figure 1 Modified diabetes prevention program consort diagram.

were male $(\mathrm{OR}=2.40 ; 95 \%$ CI 1.32 to 4.40$)$, had lower baseline BMI (OR=1.03; 95\% CI 1.01 to 1.07$)$, attended more core sessions ( $\mathrm{OR}=1.33 ; 95 \%$ CI 1.11 to 1.58$)$, and more frequently reported $(\mathrm{OR}=3.84 ; 95 \%$ CI 1.05 to $14.13)$ or met their daily fat gram $(\mathrm{OR}=4.26 ; 95 \%$ CI 1.71 to 10.57$)$ or met their weekly activity goals $(\mathrm{OR}=2.46 ; 95 \%$ CI 1.06 to 5.71) were more likely to meet their $7 \%$ weight loss goal. Treatment group, that is, urban or rural, was not a significant predictor for meeting weight loss goals
$(\mathrm{OR}=0.73 ; 95 \%$ CI 0.42 to 1.28$)$. In contrast to Rural-12, the number of participants per site was not a predictor of success.

\section{Rural- 12 analysis}

The association between covariates and outcomes in the single condition Rural-12 was also examined to determine if a shorter 12-week intervention performed similarly to the 16-week intervention. Overall, Rural-12 groups did 
Table 1 Baseline participant characteristics $(n=667)$

\begin{tabular}{|c|c|c|c|}
\hline & Urban-16 & Rural-16 & Rural-12 \\
\hline Variable & $(n=278,41.7 \%)$ & $\begin{array}{l}(n=191, \\
28.6 \%)\end{array}$ & $\begin{array}{l}(n=198, \\
29.7 \%)\end{array}$ \\
\hline $\begin{array}{l}\text { Age, mean } \\
\text { years } \pm S D\end{array}$ & $55.7 \pm 12.4$ & $55.3 \pm 12.6$ & $51.8 \pm 13.1$ \\
\hline $\begin{array}{l}\text { Weight, mean } \\
\mathrm{kg} \pm \mathrm{SD}\end{array}$ & $101.7 \pm 24.0$ & $103.6 \pm 22.6$ & $104.1 \pm 24.6$ \\
\hline BMI, mean $\mathrm{kg} / \mathrm{m}^{2} \pm \mathrm{SD}$ & $37.0 \pm 7.7$ & $37.6 \pm 7.5$ & $37.4 \pm 8.1$ \\
\hline Female, n (\%) & $231(83.1)$ & $168(88.0)$ & $171(86.4)$ \\
\hline Number of sites* & 1 & 9 & 14 \\
\hline
\end{tabular}

*Site, Montana locations for which the program was implemented. Eight sites overlapped. One site only participated.

BMI, body mass index. not perform as well as the Rural-16 and Urban-16 groups on the primary outcome measure of $7 \%$ overall weight loss $(p<0.01)$. However, a subgroup analysis found two sites performed as well as the Rural-16 and Urban-16 groups when analyzed separately with $40 \%$ of participants meeting their $7 \%$ weight loss goal and $43 \%$ of participants meeting their $5 \%$ goal. Predictors of meeting the $7 \%$ weight loss goal included greater enrollment at a participant's site, and more frequently reporting and meeting daily fat gram and weekly activity goals.

\section{Sustainability of weight loss goals}

Intervention sustainability was addressed by evaluating the retention rates within each DPP intervention and continued achievement of weight loss goals in the

Table 2 Effectiveness of modified DPP program: program participation, lifestyle change, and weight loss

\begin{tabular}{|c|c|c|c|}
\hline Variable & Urban-16 & Rural-16 & Rural-12 \\
\hline \multicolumn{4}{|c|}{ Intent-to-treat: all enrolled participants } \\
\hline \multicolumn{4}{|l|}{ Program sessions (n) } \\
\hline Total core sessions provided & 16 & 16 & 12 \\
\hline Total postcore sessions provided & 6 & 6 & 6 \\
\hline \multicolumn{4}{|l|}{ Program participation (mean $\pm S D$ ) } \\
\hline Core sessions attended & $11.4+4.3$ & $10.8+4.7$ & $7.8+3.2$ \\
\hline Postcore sessions attended & $1.6+2.0$ & $1.4+1.8$ & $1.8+2.2$ \\
\hline Total sessions attended & $13.0+5.7$ & $12.2+6.0$ & $9.6+4.9$ \\
\hline \multicolumn{4}{|c|}{ Lifestyle activities (mean $\%$ time \pm SD) } \\
\hline Daily fat gram goal met & $59.5 \pm 40.3$ & $60.1 \pm 38.9$ & $45.1 \pm 40.8$ \\
\hline Daily fat gram intake reported & $37.6 \pm 28.6$ & $42.7 \pm 31.2$ & $39.2 \pm 29.4$ \\
\hline Weekly activity goal met & $51.4 \pm 40.8$ & $47.4 \pm 38.8$ & $39.2 \pm 39.7$ \\
\hline Weekly activity minutes reported & $25.8 \pm 23.8^{*}$ & $35.1 \pm 27.8^{*}$ & $27.0 \pm 23.0$ \\
\hline \multicolumn{4}{|l|}{ Weight loss outcomes } \\
\hline$\%$ Weight loss (mean \pm SD) & $6.2 \pm 5.3$ & $5.8 \pm 5.0$ & $3.6 \pm 3.3$ \\
\hline Weight loss (mean $\mathrm{kg} \pm \mathrm{SD}$ ) & $6.2 \pm 5.8$ & $6.0 \pm 5.5$ & $3.7 \pm 3.7$ \\
\hline Participants met: & $93(33.5)$ & $66(34.6)$ & $18(18.2)$ \\
\hline $7 \%$ weight loss goal overall & $138(49.6)$ & 89 (46.6) & $43(21.7)$ \\
\hline $5 \%$ weight loss goal overall & $83(29.9)$ & $59(30.9)$ & $24(12.1)$ \\
\hline $7 \%$ weight loss goal during core & $132(47.5)$ & $85(44.5)$ & $43(21.7)$ \\
\hline \multicolumn{4}{|l|}{$5 \%$ weight loss goal during core } \\
\hline \multicolumn{4}{|l|}{ Participants completing program $\dagger$} \\
\hline \multicolumn{4}{|l|}{ Weight loss outcomes } \\
\hline$\%$ Weight loss (mean \pm SD) & $8.8 \pm 5.6$ & $8.9 \pm 4.8$ & $5.1 \pm 3.8$ \\
\hline Weight loss (mean kg $\pm \mathrm{SD}$ ) & $7.9 \pm 6.1$ & $8.0 \pm 5.1$ & $4.7 \pm 3.9$ \\
\hline Participants met & $93(46.2)$ & $66(52.1)$ & $18(25.7)$ \\
\hline $7 \%$ weight loss goal overall & $138(65.8)$ & 89 (70.3) & $43(28.7)$ \\
\hline $5 \%$ weight loss goal overall & $83(41.3)$ & $59(46.3)$ & $24(16.9)$ \\
\hline $7 \%$ weight loss goal during core & $132(65.2)$ & $85(66.9)$ & $43(28.7)$ \\
\hline $5 \%$ weight loss goal during core & & & \\
\hline
\end{tabular}

${ }^{*} \mathrm{P}<0.05$, Urban-16 compared with Rural-16 only. Due to program length differences, Rural-12 data were not included in comparison. †Completion of program requirements: core-attended one of the last three sessions; postcore-attended at least one of six sessions. DPP, diabetes prevention program. 


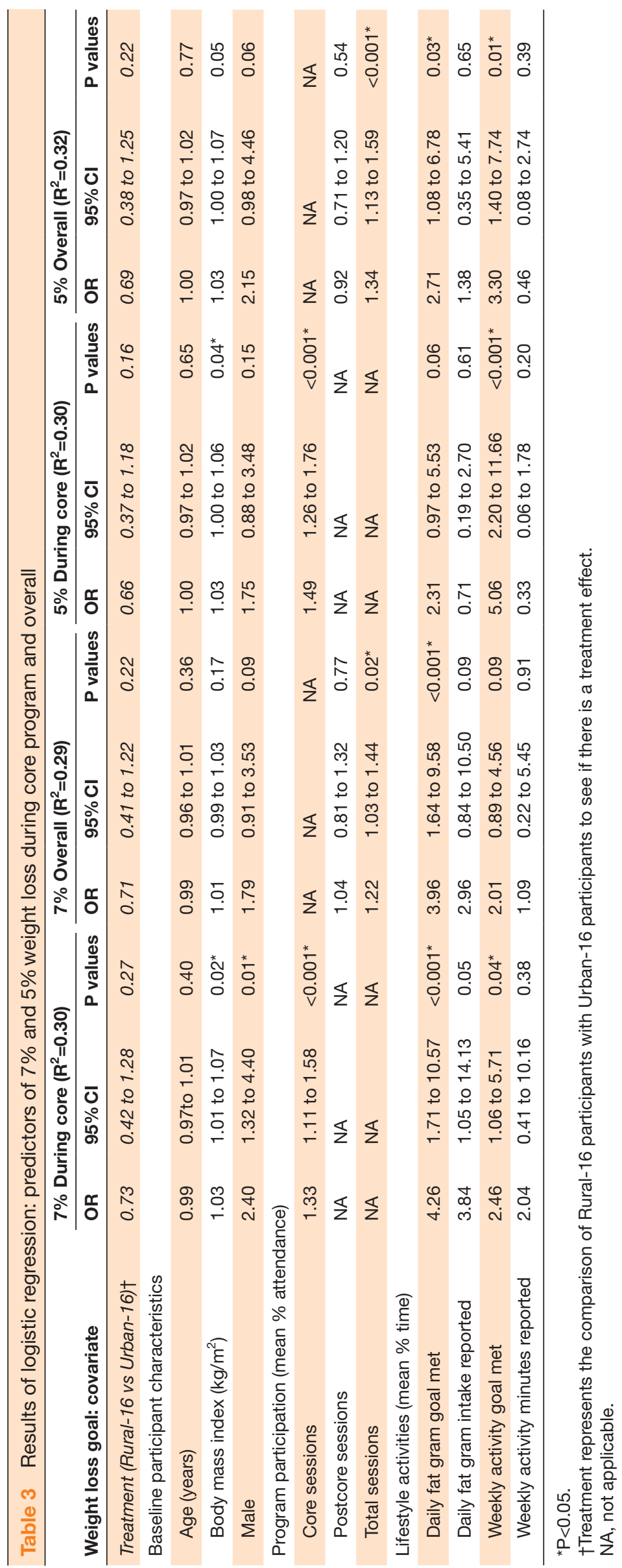


postcore period. As reported in figure 1, of the $63 \%-66 \%$ of participants who completed the core period classes, $82 \%-85 \%$ completed the postcore period. Of the 76 Urban-16 participants who met their $7 \%$ weight loss goal during the core period, $75 \%$ maintained that goal through the postcore period. Of the 56 Rural-16 participants who met the same goal, $74 \%$ maintained the goal through the postcore period. Of the 23 Rural-12 participants who met their $7 \%$ weight loss goal in the core period, $86 \%$ maintained it through the postcore period. A subset of participants who had not met their weight loss goal during the core period achieved it in the postcore period (Urban-16, 12\%; Rural-16, 18\%; Rural-12, 15\%).

\section{CONCLUSIONS}

This study demonstrates that a 16-week modified group DPP can be successfully administered to multiple rural communities simultaneously using telehealth technology with results comparable to those of an urban face-toface intervention. As hypothesized, results did not differ between the urban and telehealth groups. This is the first study that uses a telehealth delivery platform to provide a modified group-based DPP simultaneously to multiple rural communities from an urban medical hub. This study adhered to an ITT approach, providing a true indication of success among the general population.

Weight loss outcomes for this 16-week modified group DPP study compared favorably with other previously reported urban and rural modified DPP intervention studies. Compared with other 16-week DPP studies that used an ITT approach, this study showed better ${ }^{14} 162627$ or comparable $8101528-30$ results. Similar findings were found when weight loss outcomes from participants who completed the program were compared with those from urban and rural 16-week modified DDP intervention studies that only reported on participants who completed their respective programs. ${ }^{67911203132}$

Several urban and rural studies examined the percent of participants who achieved $\geq 5 \%$ or $\geq 7 \%$ weight loss and findings from this study were comparable. ${ }^{6-8} 102932$ Compared with two studies of the same telehealth DPP that delivered to one remote site simultaneous with one face-to-face group, ${ }^{8} 15$ Rural-16 participants from this study achieved similar $\geq 7 \%$ weight loss $(34.6 \%$ vs $38 \%)$, but lower $\geq 5 \%$ weight loss ( $46.6 \%$ vs $56 \%$ ).

In this study, predictors of achieving weight loss goals were comparable to those found in other studies. These included male gender, ${ }^{29}$ lower baseline BMI, ${ }^{28} 29$ and measures of monitoring fat grams ${ }^{629}$ and physical activity. ${ }^{629}$ In contrast to other studies, ${ }^{6}{ }^{28}$ age was not a predictor of meeting weight loss goals in this study at the multivariable level. Across groups, patient participation was strongly associated with intervention success. Other studies and reviews have found similar results. ${ }^{23262833}$ In a systematic review by Ali et $a l,{ }^{33}$ it was stated that with each additional lifestyle session attended, weight loss increased by 0.26 percentage points. Motivating participants to attend and engage themselves in the activities associated with the program, for example, monitoring fat grams and activity, generated success in our study and others, ${ }^{23262833}$ regardless of mode of administration, that is, face-to-face or telehealth, and may be the key to successful lifestyle interventions. In addition, this study showed that some participants did not achieve success until the postcore period, further substantiating the need for longer programs. Other studies have yet to report on sustainability of weight loss between the core and postcore periods.

A 12-week telehealth DPP was implemented and evaluated to determine if a shorter program could be as effective at rural sites as a 16-week program. A shorter program could potentially reduce cost and staff need at the urban medical hub and rural clinics. It would also decrease the time needed for telehealth connection. The evaluation of the Rural-12 intervention demonstrated mixed results. Overall, the intervention demonstrated lower success rates than the Rural-16 or Urban-16 interventions in terms of meeting weight loss goals. Compared with two ITT studies of shorter 12-week DPP programs, results from the Rural-12 intervention were better or comparable with $18.2 \%$ of participants achieving $\geq 7 \%$ weight loss (vs $13.0 \%{ }^{34}$ and $23.8 \%$ ). ${ }^{13}$ Participants at two Rural-12 communities demonstrated similar results to those participating in Rural-16 suggesting that effectiveness may be site specific and may depend on local factors such as site coordinator engagement, participant motivation, and local resources, for example, access to local exercise facilities. Successful Rural-12 sites demonstrated similar results on self-reported patient process measures, for example, self-reported diet and physical activity. Study findings do not support use of a shorter telehealth DPP if possible and instead support programs even longer than the 16-week core program.

A limitation of the study included lack of a rural faceto-face control group. This was beyond the scope and budget of this study. The results that were restricted to patients who completed the intervention were comparable to other studies that implemented face-to-face modified DPPs in rural areas. ${ }^{6-8} 152935$ In general, vascular risk factors were not directly measured in this study. Fasting blood glucose (FBG) was measured in a subset of Rural-16 participants during one intervention period with improvements similar to those found in the Urban-16 intervention (data not provided). However, it was cost-prohibitive to test FBG during subsequent sessions. Since weight loss results were similar to those studies that found successful improvement in vascular risk factors, ${ }^{9} 121316232734$ one could expect a similar beneficial effect on vascular risk factors in this study population. A cost-effectiveness analysis was also beyond the scope of this study. However, to maximize cost-effectiveness while maintaining class manageability, the authors recommend an ideal class size of about 50 participants. Another potential limitation was the fact that the teams delivering the rural and urban programs were not all 
the same people, although significant efforts were made to maintain program fidelity between treatment groups including frequent communication and consultation between the two lead dietitians.

Findings from this study add to the growing literature of how telehealth technology can be successfully used to treat obesity ${ }^{35}$ and diabetes ${ }^{818193536}$ in remote rural areas where there is a shortage of team-based obesity, diabetes and self-management support services. By conducting an ITT analysis, the authors provide a true indication of program success when delivered in a community setting. Telehealth DPP may be an effective way to prevent or delay diabetes disease progression and decrease vascular risk among at-risk individuals with obesity or overweight who otherwise would not have access to such services. This model, which links multiple rural, underserved sites simultaneously to a single urban healthcare organization DPP 'hub,' may be a more cost-effective way for health care organizations with a large rural network to provide this evidence-based program to at-risk patients in rural clinics than hiring staff to deliver the program on-site or paying qualified individuals to travel to rural communities on a weekly basis. Additionally, current advances in technology make telehealth an even less expensive option than it was at the time of this study. This model would also enable rural clinics with minimal resources to provide effective care to overweight and obese rural patients at risk for developing diabetes and other vascular disease. Finally, this study suggests that the CMS' rule on reimbursement for the DPP that went into effect in April 2018 should potentially be extended to cover telehealth-delivered DPPs. Currently, lack of reimbursement may be a barrier to dissemination of the DPP using telehealth technology.

Acknowledgements The authors thank the following individuals and organizations for their contributions to the Lifestyle Balance Telehealth Program: Eastern Montana Telemedicine Network (EMTN) staff; Center for Diabetes, Endocrinology and Metabolism staff; and especially the site coordinators and study participants of the various classes whose participation made the study possible.

Contributors ELC and PJC contributed to study design, project oversight, data analyses and writing the manuscript. BLH led all program activities and reviewed and edited the manuscript. EJM contributed to the discussion, draft and editing of the manuscript. NCC conducted all statistical analyses. WND provided oversight for all statistical analyses and reviewed the manuscript. ELC and PJC are the guarantors of this work and, as such, had full access to all the data in the study and take responsibility for the integrity of the data and the accuracy of the data analysis.

Funding Funding for this study was provided by the US Department of Health and Human Services' Health Resources and Services Administration's (HRSA) Office for the Advancement of Telehealth (OAT) (Grants: 1 H2AIT16620-01-00; 5 H2AIT16620-02-00; and 5 H2AIT16620-03-00), the Montana IDeA Network of Biomedical Research Excellence (INBRE) (Grant G129-15-W4874). The project that contributed the urban data was supported by the State of Montana and by the Grant or Cooperative Agreement Number, NU58DP004818, funded by the Centers for Disease Control and Prevention.

Disclaimer The contents of this manuscript are solely the responsibility of the authors and do not necessarily represent the official views of the Centers for Disease Control and Prevention, the Department of Health and Human Services, or Montana INBRE.

Competing interests None declared.

Patient consent Not required.
Ethics approval Billings Clinic IRB.

Provenance and peer review Not commissioned; externally peer reviewed. Data sharing statement No additional unpublished data from the study are available.

Open Access This is an Open Access article distributed in accordance with the Creative Commons Attribution Non Commercial (CC BY-NC 4.0) license, which permits others to distribute, remix, adapt, build upon this work non-commercially, and license their derivative works on different terms, provided the original work is properly cited and the use is non-commercial. See: http://creativecommons.org/ licenses/by-nc/4.0/

(c) Article author(s) (or their employer(s) unless otherwise stated in the text of the article) 2018. All rights reserved. No commercial use is permitted unless otherwise expressly granted.

\section{REFERENCES}

1. Diabetes Prevention Program Research Group. Hypertension, insulin, and proinsulin in participants with impaired glucose tolerance. Hypertension 2002;40:679-86.

2. Knowler WC, Barrett-Connor E, Fowler SE, et al. Reduction in the incidence of type 2 diabetes with lifestyle intervention or metformin. N Engl J Med 2002;346:393-403.

3. The Centers for Medicare \& Medicaid Services. Proposed policies for the Medicare diabetes prevention program expanded model in the calendar year 2018 physician fee schedule proposed rule [press release]. Baltimore, MD: The Centers for Medicare \& Medicaid Services, 2017.

4. Ratner R, Goldberg R, Haffner S, et al. Impact of intensive lifestyle and metformin therapy on cardiovascular disease risk factors in the diabetes prevention program. Diabetes Care 2005;28:888-94.

5. Orchard TJ, Temprosa M, Goldberg R, et al. The effect of metformin and intensive lifestyle intervention on the metabolic syndrome: the Diabetes Prevention Program randomized trial. Ann Intern Med 2005;142:611-9.

6. Amundson HA, Butcher MK, Gohdes D, et al. Translating the diabetes prevention program into practice in the general community: findings from the Montana Cardiovascular Disease and Diabetes Prevention Program. Diabetes Educ 2009;35:209-10.

7. Vanderwood KK, Hall TO, Harwell TS, et al. Implementing a statebased cardiovascular disease and diabetes prevention program. Diabetes Care 2010;33:2543-5.

8. Vadheim LM, McPherson C, Kassner DR, et al. Adapted diabetes prevention program lifestyle intervention can be effectively delivered through telehealth. Diabetes Educ 2010;36:651-6.

9. Ackermann RT, Finch EA, Brizendine E, et al. Translating the Diabetes Prevention Program into the community. The DEPLOY Pilot Study. Am J Prev Med 2008;35:357-63.

10. Brokaw SM, Carpenedo D, Campbell P, et al. Montana Cardiovascular Disease and Diabetes Prevention Workgroup. Effectiveness of an adapted diabetes prevention program lifestyle intervention in older and younger adults. J Am Geriatr Soc 2015;63:1067-74.

11. Dallam GM, Foust CP. A comparative approach to using the diabetes prevention program to reduce diabetes risk in a worksite setting. Health Promot Pract 2013;14:199-204.

12. Katula JA, Vitolins MZ, Rosenberger EL, et al. One-year results of a community-based translation of the Diabetes Prevention Program: Healthy-Living Partnerships to Prevent Diabetes (HELP PD) Project. Diabetes Care 2011;34:1451-7.

13. Kramer MK, Kriska AM, Venditti EM, et al. Translating the Diabetes Prevention Program: a comprehensive model for prevention training and program delivery. Am J Prev Med 2009;37:505-11.

14. McTigue KM, Conroy MB, Hess R, et al. Using the internet to translate an evidence-based lifestyle intervention into practice. Telemed J E Health 2009;15:851-8.

15. Vadheim LM, Patch K, Brokaw SM, et al. Telehealth delivery of the diabetes prevention program to rural communities. Trans/ Behav Med 2017;7:286-91.

16. Boltri JM, Davis-Smith YM, Seale JP, et al. Diabetes prevention in a faith-based setting: results of translational research. J Public Health Manag Pract 2008;14:29-32.

17. Vadheim LM, Brewer KA, Kassner DR, et al. Effectiveness of a lifestyle intervention program among persons at high risk for cardiovascular disease and diabetes in a rural community. $J$ Rural Health 2010;26:266-72. 
18. Siminerio L, Ruppert K, Huber K, et al. Telemedicine for Reach, Education, Access, and Treatment (TREAT): linking telemedicine with diabetes self-management education to improve care in rural communities. Diabetes Educ 2014;40:797-805.

19. Ciemins E, Coon P, Peck R, et al. Using telehealth to provide diabetes care to patients in rural Montana: findings from the promoting realistic individual self-management program. Telemed $\mathrm{J}$ E Health 2011;17:596-602.

20. Neamah HH, Sebert Kuhlmann AK, Tabak RG. Effectiveness of Program Modification Strategies of the Diabetes Prevention Program: A Systematic Review. Diabetes Educ 2016;42:153-65.

21. Diabetes Prevention Program Research Group. Diabetes prevention program outcomes study. Lifestyle manuals of operations: group lifestyle balance materials: University of Pittsburgh. 2011 https:// dppos.bsc.gwu.edu/web/dppos/lifestyle (Cited 30 Nov 2011).

22. Center for Disease Control and Prevention. Center for Disease Control and Prevention Diabetes Prevention Recognition Program Standards and Operating Procedures. 2018 www.cdc.gov/diabetes/ prevention/recognition (Cited 1 Mar 2018).

23. Dunkley AJ, Bodicoat DH, Greaves CJ, et al. Diabetes prevention in the real world: effectiveness of pragmatic lifestyle interventions for the prevention of type 2 diabetes and of the impact of adherence to guideline recommendations: a systematic review and meta-analysis. Diabetes Care 2014;37:922-33.

24. Nagelkerke NJD. A note on a general definition of the coefficient of determination. Biometrika 1991;78:691-2.

25. Tabachnick BG, Fidell LS. Using multivariate statistics. 6th ed. Boston, MA: Pearson, 2013.

26. Ackermann RT, Liss DT, Finch EA, et al. A Randomized Comparative Effectiveness Trial for Preventing Type 2 Diabetes. Am J Public Health 2015;105:2328-34.
27. Weinhold KR, Miller CK, Marrero DG, et al. A Randomized Controlled Trial Translating the Diabetes Prevention Program to a University Worksite, Ohio, 2012-2014. Prev Chronic Dis 2015;12:E210.

28. Brokaw SM, Arave D, Emerson DN, et al. Intensive lifestyle intervention goals can be achieved as effectively with large groups as with small groups. Prim Care Diabetes 2014;8:295-300.

29. Harwell TS, Vanderwood KK, Hall TO, et al. Factors associated with achieving a weight loss goal among participants in an adapted Diabetes Prevention Program. Prim Care Diabetes 2011;5:125-9.

30. Kramer MK, Kriska AM, Venditti EM, et al. A novel approach to diabetes prevention: evaluation of the Group Lifestyle Balance program delivered via DVD. Diabetes Res Clin Pract 2010;90:e60-3.

31. McTigue KM, Conroy MB, Bigi L, et al. Weight loss through living well: translating an effective lifestyle intervention into clinical practice. Diabetes Educ 2009;35:199-204.

32. Michaelides A, Raby C, Wood M, et al. Weight loss efficacy of a novel mobile diabetes prevention program delivery platform with human coaching. BMJ Open Diabetes Res Care 2016;4:e000264.

33. Ali MK, Echouffo-Tcheugui J, Williamson DF. How effective were lifestyle interventions in real-world settings that were modeled on the Diabetes Prevention Program? Health Aff 2012;31:67-75.

34. Sattin RW, Williams LB, Dias J, et al. Community trial of a faithbased lifestyle intervention to prevent diabetes among AfricanAmericans. J Community Health 2016;41:87-96.

35. Harvey-Berino J. Changing health behavior via telecommunications technology: using interactive television to treat obesity. Behav Ther 1998;29:505-19.

36. Holloway B, Coon PJ, Kersten DW, et al. Telehealth in rural montana: promoting realistic independent self-management of diabetes. Diabetes Spectrum 2011;24:50-4. 